\title{
Modelo de la tensión inducida por un rayo sobre líneas de distribución
}

\author{
V. Henao - Céspedes ${ }^{\# 1}$, L.F Díaz - Cadavid ${ }^{\# 2}$, W.G Fano ${ }^{\# 3}$, E.A Cano - Plata ${ }^{\# 4}$ \\ \# 1 Universidad Nacional de Colombia - Universidad Católica de Manizales \\ 1 vhenaoceunal.edu.co \\ \# 2 Universidad Nacional de Colombia \\ lfdiazc@unal.edu.co \\ \# 3 Universidad de Buenos Aires \\ 3 gustavo.fanodieee.org \\ \# 4 Universidad Nacional de Colombia \\ ${ }^{4}$ eacanopleunal. edu.co \\ Recibido: 28/02/17; Aceptado: 20/08/17
}

\begin{abstract}
In this work the electromagnetic emissions caused by a discharge of a lightning have been modeled, considering the lightning as a short monopole antenna over a perfectly conducting ground plane. By this analogy the radiated electric and magnetic fields are calculated, and then the induced voltage is calculated in a distribution line considered as a short loop.

The mathematical expression used for the calculation of the induced voltage was already proposed in a previous work and in the present work is validated by its confrontation in an simulated scenario with two known expressions for the calculation of the induced voltage, one recommended by the IEEE and developed by S. Rusck and another developed by Barbosa.

On the basis of the considerations made by Rusck to obtain its expression for the induced voltage, the geometry related to the proposed expression is adapted to make it comparable with Rusck's international reference in the IEEE standard.

Later, the $d V_{\text {ind }} / d y$ behaviors of the three expressions in the region of near-field radiation of the lightning were analyzed, determining that the trends (which are hyperbolic) of the proposed expression and that developed by Rusck (internationally standardized in the IEEE 1410 standard) have a great similarity when the radiated fields exceed $20 \mathrm{~m}$, giving validity to the proposed expression.
\end{abstract}

Resumen - En este trabajo se han modelado las emisiones electromagnéticas ocasionadas por la descarga de un rayo, considerando al rayo como una antena monopolo corto sobre un plano de tierra perfectamente conductor. Mediante esta analogía se calculan los campos eléctricos y magnéticos radiados, y luego se calcula la tensión inducida en una línea de distribución considerada como un lazo corto.

La expresión matemática utilizada para el cálculo de la tensión inducida ya fue propuesta en un trabajo previo y en el presente trabajo es validada mediante su confrontación en un escenario simulado con dos expresiones conocidas para el cálculo de la tensión inducida, una recomendada por la IEEE y desarrollada por S. Rusck y otra desarrollada por Barbosa.

Sobre la base de las consideraciones realizadas por Rusck para la obtención de su expresión para la tensión inducida, la geometría relacionada con la expresión propuesta es adaptada para hacerla comparable con el referente internacional de Rusck en la norma IEEE.

Posteriormente se han analizado los comportamientos $d V_{\text {ind }} / d y$ de las tres expresiones en la región de radiación de campo cercano del rayo, determinando que las tendencias (las cuales son de carácter hiperbólico) de la expresión propuesta y la desarrollada por Rusck (estandarizada a nivel internacional en la norma IEEE 1410) presentan una gran similitud cuando los campos radiados sobrepasan los $20 \mathrm{~m}$, dando validez a la expresión propuesta.

\section{INTRODUCCIÓN}

En la región norte de Argentina, en limites con Brasil se encuentra una zona con un nivel ceráunico (NC) de 70 días tormentosos por año [1], siendo dicha zona la de más alto $\mathrm{NC}$ en Argentina. En contraste con Colombia, donde por su ubicación geográfica en la zona de convergencia intertropical (ZCI) se encuentran regiones al centro del país con NC de 140 días tormentosos por año [2]. Si bien lo anterior indica que definir la posible responsabilidad del rayo en la ocurrencia de fallas sobre la línea de distribución es más importante en Colombia, no se puede descartar que por los cambios climáticos a final del siglo se puede ver incrementada la actividad atmosférica en un 50\% según [3], lo cual se vería reflejado en países como Argentina donde se comenzaría a requerir estudios que definan la responsabilidad del rayo en la ocurrencia de fallas en líneas de distribución.

Un parámetro de suma importancia para definir la posible responsabilidad del rayo en la ocurrencia de fallas sobre la línea de distribución es la tensión inducida ( $\left.\mathbf{V}_{\text {ind }}\right)$ por el rayo, entre las líneas de distribución eléctrica que conforman un sistema bifilar y tierra. Una expresión matemática para estimar $\mathbf{V}_{\text {ind }}$ ha sido desarrollada en trabajos previos [4], dicha expresión fue obtenida desde una perspectiva basada en la aplicación de la teoría de la radio propagación y de los sistemas radiantes, y modelando a la línea de distribución como una antena tipo lazo corto (Fig. 1). 


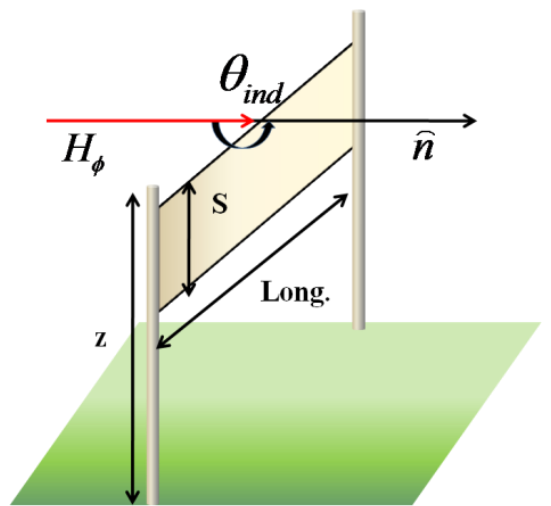

Fig. 1. Línea de distribución vista como una antena lazo corto

Según S. Rusck [5] si la tensión inducida es mayor que el nivel de aislamiento de la línea ${ }^{1}$ (que se supone es igual en toda la línea), un flameo ${ }^{2}$ (corriente de falla) puede ocurrir, lo cual se vería reflejado como una falla en el suministro eléctrico. Es por ello, que atendiendo a Rusck, se requiere validar la expresión propuesta en [1] para el cálculo del valor $\mathbf{V}_{\text {ind, }}$ siendo esta:

$$
V_{\text {ind }}=\frac{f_{n} \mu I_{n} L \sin \theta \cos \theta_{i n d} A e^{(-j \beta r)}}{4}\left(\frac{1}{r^{2}}+\frac{j \beta}{r}\right)
$$

Donde:

fn: es la frecuencia de la n-ésima componente espectral del rayo.

$\boldsymbol{\mu}$ : permeabilidad magnética de la línea de distribución.

In: corriente máxima de la n-ésima componente espectral. L: altura del rayo (Entre nube y tierra).

$\boldsymbol{\theta}_{\text {ind: ángulo de inducción del campo magnético acimutal }}$ respecto a la normal $(\widehat{n})$ de la línea de distribución ver (Fig. 1).

A: Área encerrada por el lazo ver (Fig. 1).

$\boldsymbol{\beta}$ : relación entre la velocidad de la descarga de retorno y la velocidad de la luz.

Para validar la expresión (1) propuesta se plantea un escenario virtual (modelado) que permita mediante una simulación computacional establecer un marco comparativo entre los resultados de la aplicación de dicha expresión y los que se obtienen de aplicar a ese mismo escenario dos expresiones conocidas para el cálculo de la tensión inducida.

La primera de las expresiones recomendadas es sugerida por la norma IEEE 1410 [6] y desarrollada por S. Rusck [5] para calcular la tensión inducida en el punto más cercano sobre la línea de distribución al sitio del impacto del rayo a partir del conocimiento del potencial escalar. Esta expresión al estar sugerida en una norma IEEE permite contar con un referente estandarizado y de uso internacional para la validación. La expresión en mención es:

\footnotetext{
${ }^{1}$ Nivel de aislamiento: Establece el valor máximo de tensión que pueden soportar los equipos conectados a la línea sin que se produzca flameo. ${ }^{2}$ Flameo: Arco eléctrico
}

$$
V_{\text {peak }}=\frac{Z_{o} I_{o} h}{y}\left(1+\left(\frac{1}{\sqrt{2}} \frac{v}{c} \frac{1}{\sqrt{1-\frac{1}{2}\left(\frac{v}{c}\right)^{2}}}\right)\right)
$$

Donde:
$\mathbf{Z}_{\mathbf{0}}=377 \Omega$ (Impedancia intrínseca del aire),
$\mathbf{I}_{\mathbf{o}}$ : es la corriente pico del rayo
h: es la altura promedio de la línea
y: es la distancia horizontal más cercana entre la línea y
$\boldsymbol{v}$ : es la velocidad de la descarga de retorno
c: es la velocidad de la luz el rayo

La segunda expresión a confrontar es desarrollada por Barbosa en [7], y permite calcular la tensión inducida por el rayo a partir del campo eléctrico debido al potencial vectorial en un lazo corto. Considerando que la expresión (1) propuesta calcula la tensión inducida sobre la línea de distribución modelada como una antena lazo corto, reviste especial interés poder validar los resultados de esta con los obtenidos con la propuesta por Barbosa [7], teniendo así un nuevo punto de comparación. Esta segunda expresión se formula como:

$$
V_{R}(t)=2 \times 10^{-7} h \frac{I_{0}}{T} \ln \left\{\frac{r_{2}\left[v t+\sqrt{(v t)^{2}+\lambda r_{1}^{2}}\right]}{r_{1}\left[v t+\sqrt{(v t)^{2}+\lambda r_{2}^{2}}\right]}\right\}
$$

Donde:

Io: es la corriente pico del rayo

$\boldsymbol{v}$ : es la velocidad de la descarga de retorno

$\lambda=1-v_{R}^{2}$

$v_{R}=v / c$

r1: es la distancia menor entre el lazo y el rayo

r2: es la distancia mayor entre el lazo y el rayo

\section{FUNDAMENTOS DEL MODELO}

En el presente trabajo el rayo es asumido como un monopolo corto, considerando que una antena se define como pequeña (corta) si su dimensión más grande, no excede un decimo de la longitud de onda $(\lambda / 10)$ de la frecuencia en la cual está operando [8] siendo este nuestro caso. Por otro lado, según la teoría de imágenes explicada por Schelkunoff en [8] y para efectos de cálculo, la antena rayo (monopolo) se tratará inicialmente como un dipolo corto efectuando los ajustes respectivos.

Asimismo con el fin de efectuar el modelo propuesto se tiene en cuenta que:

1. Conforme la Ley de Faraday para que se produzca una inducción electromagnética la corriente debe ser variable, de esta manera se explica que la variación de corriente del rayo en el tiempo es la responsable de que se genere una inducción sobre los conductores que son incididos por los 
campos electromagnéticos (CEM) emitidos por este. La anterior premisa indica a su vez que la corriente continua (CC) no induce, puesto que no representa una variación temporal y por tanto la componente espectral asociada a la $\mathrm{CC}$ del rayo no se tiene en cuenta.

2. En el Grupo de Investigación en Telemática y Telecomunicaciones (GTT) el cual lidera esta investigación, se desarrolló un sistema para registrar y analizar espectralmente las descargas atmosféricas, dicho sistema llamado LEMPSA - 1 fue elaborado con tecnología SDR (Software Defined Radio) [9]. El sistema LEMPSA - 1 permitió confirmar que el pulso electromagnético generado por el rayo (LEMP - Lightning Electromagnetic Pulse) emite señales con alta concentración de energía espectral en la banda cercana o centrada en los $10 \mathrm{kHz}$, lo que indica que la longitud de onda de la señal LEMP emitida por el rayo para esta frecuencia se estima cercana a los $30 \mathrm{~km}$.

3. La altura promedio de un rayo nube - tierra es de 1,5 $\mathrm{km}$ [9] y el canal del rayo es completamente vertical sobre la tierra con un diámetro mucho más inferior que su altura.

En consecuencia, si es posible asumir el rayo como una antena monopolo corto [9][10], dado que el rayo visto así cumple efectivamente la condición de tener una longitud $\mathrm{L} \leq 0,05 \lambda[11]$.

Los CEM radiados por el rayo inducen la línea de distribución, que actúa entonces como una antena receptora de lazo corto y el rayo de esta manera se comporta como una antena transmisora tipo monopolo, conformando así un sistema de comunicación (Ver Fig. 2).

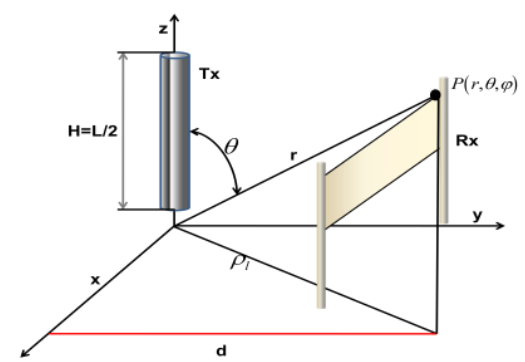

Fig. 2. Geometría para definir la tensión inducida en un punto a una distancia d.

La expresión (1) propuesta para el cálculo de $\mathbf{V}_{\text {ind, }}$ difiere de las expresiones (2) y (3) propuestas por Rusck [5] y Barbosa [7] respectivamente, en que la tensión inducida es estimada a partir de $H_{\phi_{n}}$ (Campo magnético acimutal) y las diferentes componentes espectrales del rayo, y además es calculada sobre la totalidad de la línea de distribución .

Rusck [5], [12] utiliza la distancia más cercana al punto de impacto del rayo haciendo que la abscisa sea $\mathbf{x}=\mathbf{0}$ y obtiene la tensión en un único punto sobre la línea. Por tanto a raíz de esta última consideración, el área de la expresión (1) es propuesta con tendencia a un valor infinitesimal comparable con un único punto sobre la línea. Asimismo atendiendo a Rusck [12] se asume que el campo eléctrico, el potencial vectorial y el potencial escalar son constantes e iguales entre la altura de la línea y el suelo. Por otro lado según Thottappillil [10] para considerar los campos electromagnéticos a nivel del suelo el ángulo $\theta$ toma un valor igual a $90^{\circ}$.

De tal forma que teniendo en cuenta estas consideraciones la geometría mostrada en la Fig. 2, es adaptada para hacerla comparable con la propuesta por Rusck, obteniendo de esta manera la geometría consignada en la Fig. 3 y en base a la cual se realizará la validación de la expresión (1).

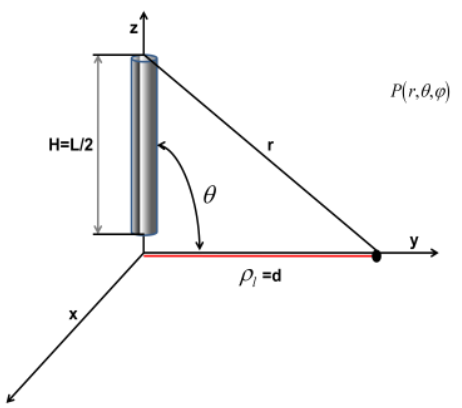

Fig. 3. Geometría modificada del rayo (monopolo) y su punto de inducción sobre la línea (lazo corto)

\section{RESULTADOS DE SIMULACIÓN}

Para el modelado del escenario virtual se plantea una línea de distribución bifilar con disposición vertical en poste (con esta disposición se garantiza que $\theta_{\text {ind }}=0$, lo que origina una máxima inducción sobre el lazo), de $13,2 \mathrm{kV}$ de media tensión (MT). La línea se estima con una altura promedio de $10 \mathrm{~m}$ con relación a la superficie del terreno. Cómo material de la línea se considera el aluminio, y por ello el valor de la permeabilidad magnética $\boldsymbol{\mu}$ es la de dicho material.

Por otro lado, teniendo en cuenta que para una misma estructura de apoyo como distancia horizontal mínima de seguridad entre conductores se recomienda para líneas de $13,2 \mathrm{kV}$ una distancia mínima de $30 \mathrm{~cm}$ más $1 \mathrm{~cm}$ por $\mathrm{kV}$ sobre $8,7 \mathrm{kV}$ [13], en el presente escenario se ha asumido una separación entre conductores de $1,3 \mathrm{~m}$ valor usado por el operador eléctrico de la región [14] en la cual se desarrolla este estudio. Una vez establecido el escenario, se simula mediante un método computacional un impacto de rayo con una forma de onda de corriente tipo Heidler con unos tiempos tomados de la norma IEEE 1410 [6] para el frente de $5,63 \mu \mathrm{s}$ y para la cola de $77,5 \mu \mathrm{s}$, con un valor pico de corriente de $42 \mathrm{kA}$ (promedio para Colombia) [15], y una altura media entre nube y tierra de $1500 \mathrm{~m}$ [9]. A la forma de onda simulada se le aplica el método de la transformada rápida de Fourier (FFT) para obtener las componentes espectrales requeridas para la simulación de la expresión (1) Modelado el escenario y aplicando las expresiones antes mencionadas, se calculó la tensión inducida a diferentes distancias de impacto. Los resultados obtenidos son graficados en la Fig. 4:

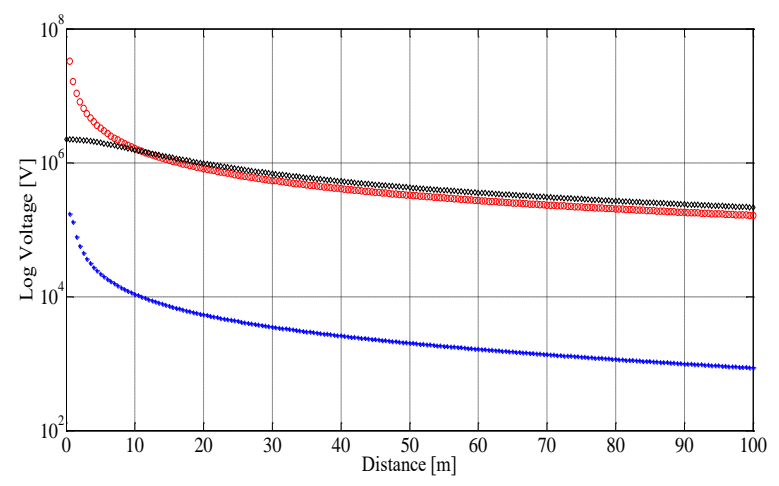

Fig. 4. Tensión inducida en función de la distancia. 
En negro la tensión inducida obtenida con (1), en rojo obtenida con (2), en azul obtenida con (3)

Para una mejor interpretación de la gráfica de la Fig. 4 esta se subdivide en dos (tramos) con lo cual se obtienen dos nuevas gráficas (Fig. 5 y Fig. 6). En la gráfica de la Fig. 5 que corresponde al primer tramo que va de 0 a $20 \mathrm{~m}$, es observada una diferencia de 27,36 \% entre los valores arrojados por la expresión propuesta y la desarrollada por Rusck.

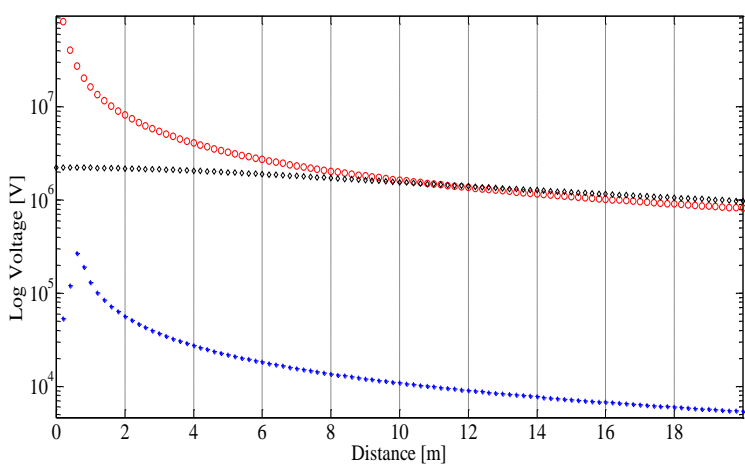

Fig. 5. Primer tramo de inducción. En negro la tensión inducida obtenida con(1), en rojo obtenida con (2), en azul obtenida con (3).

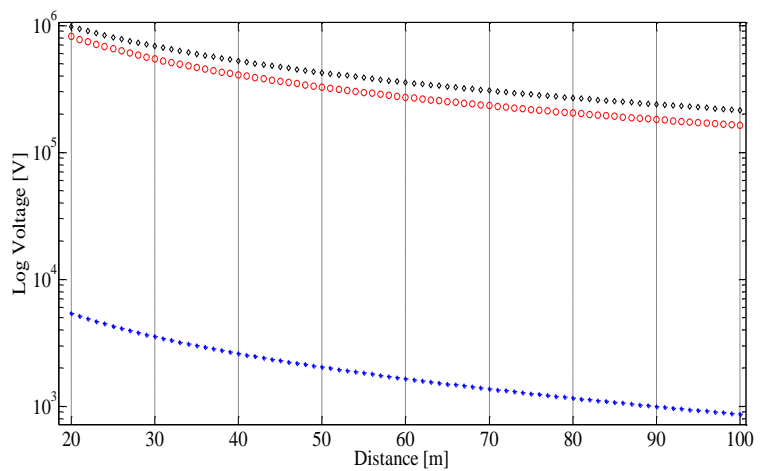

Fig. 6. Segundo tramo de inducción. En negro la tensión inducida obtenida con (1), en rojo obtenida con (2), en azul obtenida con (3).

En la gráfica de la Fig. 6 correspondiente al segundo tramo (20 m en adelante) si bien la diferencia se incrementa a 39,68\%, es posible observar una similitud en la tendencia entre la expresión propuesta y la expresión de Rusck.

Por otro lado para efectos teóricos y con el fin de determinar el comportamiento de la tensión inducida en la región de campo cercano, se establece un límite de $15 \mathrm{~km}$, considerando que según la teoría de sistemas radiantes el campo lejano del rayo está definido por un criterio según el cual el límite inferior del campo lejano $r$ debe ser $r \geq \lambda / 2$ [11], lo que en este caso es igual a $15 \mathrm{~km}$. Para este nuevo límite se realizan los cálculos de la Tensión inducida cuyos resultados se pueden apreciar en la gráfica de la Fig. 7:

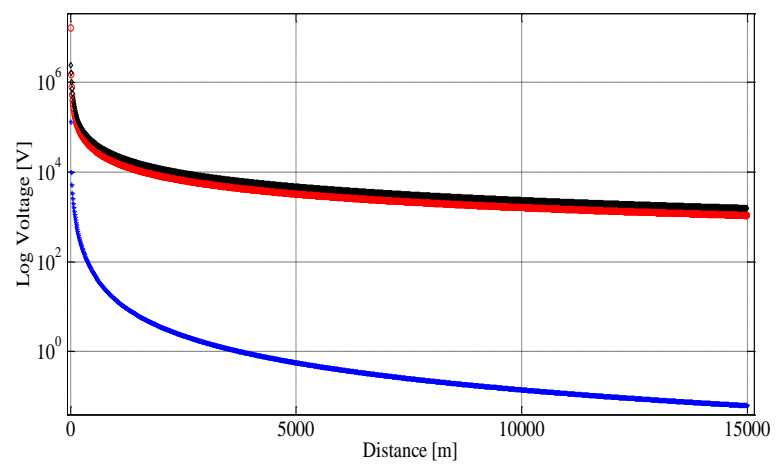

Fig. 7. Tensión inducida en la región de campo cercano. En negro la tensión inducida obtenida con (1), en rojo obtenida con (2), en azul obtenida con (3).

La gráfica de la Fig. 7 permite determinar que la expresión propuesta sigue la misma tendencia hiperbólica de la expresión desarrollada por Rusck, validando de esta manera la expresión propuesta.

\section{ANÁLISIS DE RESULTADOS}

Analizadas las figuras anteriores se puede observar que comparados los resultados obtenidos con las expresiones (1) y (2) estos son bastantes similares, con lo cual la expresión propuesta queda validada con un referente de carácter internacional sugerido por la norma IEEE 1410 [6] y desarrollado por Rusck [5].

Por otro lado, si se comparan los resultados obtenidos entre la expresión (3) y las expresiones (1) y (2) se observa que si bien son similares en forma son bastante diferentes en magnitud. Esta diferencia puede tener explicación en que en la expresión (3) propuesta y desarrollada por Barbosa [7] no se considera ni la altura del rayo, ni la permeabilidad magnética $\mu$ parámetros que si son tenidos en cuenta en la expresión propuesta. Adicionalmente la expresión (2) tiene comportamiento hiperbólico y la (1) de hiperbólico acotado por una tendencia exponencial, definiendo así la diferencia en los metros más cercanos al punto de impacto del rayo, en donde los resultados de la expresión (1) son menores que los de la (2). Al incrementar la distancia la tendencia exponencial hace que los resultados de la expresión (1) decrezcan más lentamente que los resultados de la expresión (2) que decrecen más rápidamente por la tendencia hiperbólica. A distancias mayores a los $20 \mathrm{~m}$ las dos expresiones tienden a mostrar un comportamiento $d V_{\text {ind }} / d y$ similar debido a la tendencia hiperbólica de ambas.

En otras palabras, las diferencias presentadas pueden tener su explicación en un simple hecho basado en que la expresión (1) propuesta considera la inducción sobre la totalidad de la línea de distribución, la variable permeabilidad magnética $\mu$ del material de la línea, la separación entre conductores y el uso del contenido espectral del rayo, parámetros que no son tenidos en cuenta por las expresiones (2) y (3). 


\section{CONCLUSIONES}

Se han modelado las emisiones electromagnéticas ocasionadas por la descarga de un rayo, considerando al rayo como una antena monopolo corto sobre un plano de tierra perfectamente conductor. Se calculó la tensión inducida en la línea de distribución de energía eléctrica de Media Tensión mediante una expresión analítica.

La expresión propuesta para el cálculo de la tensión inducida por el rayo en líneas de distribución ha sido validada confrontándola con las expresiones planteadas por Rusck [5] y Barbosa [7]. Dicha expresión a diferencia de los habituales modelos eléctricos, utiliza la teoría de los sistemas radiantes basándose en un análisis de la propagación de los CEM asociados al LEMP asumiendo el rayo como una antena transmisora tipo monopolo y la línea de distribución como una antena receptora tipo lazo corto.

Considerando que a diferencia de las expresiones (2) y (3) en la expresión propuesta se modela la línea de distribución como una antena lazo corto y que asimismo, se consideran las componentes espectrales del rayo además de parámetros adicionales de la línea de distribución tales como la permeabilidad magnética $\mu$ del material de la línea y la separación entre conductores, queda demostrado que la expresión propuesta es una herramienta valida en el cálculo de la tensión inducida desde la perspectiva de la teoría de la radio propagación y de los sistemas radiantes.

\section{TRABAJOS FUTUROS}

Se plantea como trabajo futuro el establecer un escenario experimental donde se modele físicamente una línea de distribución y se genere artificialmente un pulso electromagnético similar al que genera un rayo en su proceso de descarga, para de esta manera obtener mediciones de las tensiones inducidas por rayos que impactan a diferentes distancias de la línea y comparar dichas mediciones con los resultados obtenidos con la expresión matemática propuesta en el presente artículo.

\section{AGRADECIMIENTOS}

Los autores agradecen a la Universidad Nacional de Colombia Sede Manizales, a la Universidad de Buenos Aires, a la Universidad Católica de Manizales y a COLCIENCIAS.

\section{REFERENCIAS}

[1] IRAM, Norma IRAM 2427. Protección contra el impulso electromagnético generado por el rayo (“ LEMP "). Argentina, 2006.

[2] ICONTEC, NTC - 4552Protección contra rayos. Principios Generales. Colombia, 2004.

[3] D. M. Romps, J. T. Seeley, D. Vollaro, and J. Molinari, "Projected increase in lightning strikes in the United States due to global warming," vol. 346, no. 6211, pp. 851-853, 2014.

[4] V. Henao - Céspedes; W.G Fano; L.F Díaz - Cadavid; E.A Cano - Plata, "Induced voltage approach by lightning in the distribution lines," in 2016 IEEE Global Electromagnetic Compatibility Conference (GEMCCON), 2016, pp. 1-5.

[5] S. Rusck, "Protection of Distribution Lines," in Lightning Volume 2: Lightning Protection, R. H. Golde, Ed. Londin: Academic Press, 1977, pp. 747-771.

[6] IEEE Guide for Improving the Lightning Performance of Electric Power Overhead Distribution Lines. 2004.

[7] C. F. Barbosa and J. O. S. Paulino, "A Closed Expression for the Lightning Induced Voltage in Short Loops," IEEE Trans. Electromagn. Compat., 2016.

[8] S. A Schelkunoff; H. T Friss, Antennas Theory and Practice. New York: John Wiley \& Sons, Inc. London. Chapman \& Hall, Limited., 1952.

[9] L.F Diaz Cadavid, "Caracterización espectral del pulso electromagnético radiado por el rayo atmosférico (LEMP) mediante un Detector-Receptor de RF tipo SDR (SoftwareDefined Radio)," Universidad Nacional de Colombia, Sede Manizales, 2014.

[10] R. Thottappillil, "Computation of electromagnetic fields from lightning discharge," in The Lightning Flash, 2nd ed., V. Cooray, Ed. London: The Institution of Engineering and Technology, 2014, pp. 351-403.

[11] V. Trainotti; W. G. Fano; L. A. Dorado, Ingeniería electromágnetica: polarización, reflexión de ondas, radiación electromagnética, sistemas radiantes lineales. Vol. 2. Buenos Aires: Nueva Librería, 2005.

[12] F. Mottola, "Methods and techniques for the evaluation of lightning induced overvoltages on power lines. Application to MV distribution systems for improving the quality of power supply," University Federico II of Napoli, 2007.

[13] Reglamento Técnico de Instalaciones Eléctricas (RETIE). Colombia, 2013.

[14] Central Hidroeléctrica de Caldas, Manual de normas de diseño y construcción. Colombia, 2016.

[15] IEEE Power and Energy Society, IEEE Guide for the Application of Insulation Coordination. 1999. 